\title{
Initial magnetic field configurations for 3-dimensional simulations of astrophysical jets
}

\author{
M. A. S. G. Jørgensen ${ }^{1}$, R. Ouyed ${ }^{2}$, and M. Christensen ${ }^{1}$ \\ 1 University of Copenhagen, Astronomical Observatory, Juliane Maries Vej 30, 2100 Copenhagen $\varnothing$, Denmark \\ 2 Nordic Institute for Theoretical Physics, Blegdamsvej 17, 2100 Copenhagen Ø, Denmark
}

Received 27 June 2001 / Accepted 21 September 2001

\begin{abstract}
We solve, and provide analytical expressions, for current-free magnetic configurations in the context of initial setups of 3-dimensional simulations of astrophysical jets involving an accretion disk corona in hydrostatic balance around a central object. These configurations which thread through the accretion disk and its corona preserve the initial hydrostatic state. This work sets stage for future 3-dimensional jet simulations (including disk rotation and mass-load) where launching, acceleration and collimation mechanisms can be investigated.
\end{abstract}

Key words. MHD - stars: winds - outflows

\section{Introduction}

The most successful model of astrophysical jets is that of magneto-hydrodynamic (MHD) driven winds (Blandford \& Payne 1982). In this model, the magnetic field is predicted to launch, accelerate and efficiently collimate the jet. The advent of MHD codes over the last decade allowed us to study the details of this model through the use of time-dependent MHD simulations and confirm the importance of the magnetic field. One point that has been emphasized in many of these simulations is the importance of properly setting up the initial state. A numerically stable initial setup makes the simulation tractable and allows contact with theory. Here, we are particularly interested in simulations as presented in Ouyed \& Pudritz (1997a, OPI) and Ouyed \& Pudritz (1997b, OPII) focusing on the technical aspect of the initial state.

The initial conditions as defined in OPI and OPII correspond to a central object (a proto-star) surrounded by a Keplerian disk and an overlying corona in hydrostatic equilibrium. The disk has fixed properties and provides the boundary conditions for the outflow. The time evolution of the initial state is partly depicted by the momentum equation (using the standard nomenclature),

$\rho\left(\frac{\partial \boldsymbol{v}}{\partial t}+(\boldsymbol{v} \cdot \nabla) \boldsymbol{v}\right)+\nabla p+\rho \nabla \Phi-\boldsymbol{J} \times \boldsymbol{B}=0$,

and would, in general, be perturbed by the Lorentz force of a magnetic field that threads through the accretion disk and its corona. Therefore, all previous 2-dimensional (2-D)

Send offprint requests to: M. Jørgensen, e-mail: svart@astro.ku.dk simulations (OPI\&II) employ initialy current-free, $\boldsymbol{J}=0$, magnetic fields where the condition for stability becomes

$0=-\frac{\nabla p}{\rho}-\nabla \Phi$

at all time. This initial setup was successfully extended to 3-dimensional (3-D) simulations in Ouyed et al. (2001, OPC). However, the work presented in OPC was restricted to the simple case of a uniform magnetic configuration where the accretion disks is threaded by vertical field lines. In this paper, we solve for non-uniform current-free magnetic fields in 3-D which would complete our 3-D initial hydrostatic corona as defined in Eq. (2). The paper is organized as follows: in Sect. 2, we describe the force-free equations defining our problem. In Sect. 3 we solve for the current-free case and test the stability of these solutions by evolving the initial setup in time. We introduce and describe the JETSET tool developed for this purpose. We conclude in Sect. 4.

\section{Force-free configurations}

Force-free configurations are characterized by

$(\boldsymbol{J} \times \boldsymbol{B}=0, \nabla \cdot \boldsymbol{B}=0)$,

corresponding to the standard two cases, $\boldsymbol{J} \| \boldsymbol{B}$, and $\boldsymbol{J}=0$. We start with the case where currents are parallel to the magnetic field lines. Or,

$\boldsymbol{B}=\boldsymbol{\nabla} \times\left(\frac{\boldsymbol{B}}{\alpha}\right)$, 
(here, $\alpha$ is constant), i.e. the vector potential is given by $\frac{1}{\alpha} \boldsymbol{B}$ (from $\boldsymbol{B}=\boldsymbol{\nabla} \times \boldsymbol{A}$ ). Thus we first solve for the magnetic field and then calculate the vector potential; recall that in deriving $\boldsymbol{B}$ using the vector potential, $\boldsymbol{\nabla} \cdot \boldsymbol{B}=0$ is numerically guaranteed to within machine round-off errors. Here and in the rest of the paper, we adopt cylindrical coordinates $(r, \phi, z)$ and we assume that the fields are separable.

Using relation (4) and the fact that the magnetic field is divergence free we arrive at:

$\alpha^{2} \boldsymbol{B}=-\nabla^{2} \boldsymbol{B}$.

This is a vector relation and therefore also holds for every component. Let us write a component of the magnetic field, $\boldsymbol{B}$, as $B$ and making use of our usual assumption of separable variables we find:

$$
\begin{aligned}
B & =R(r) \Phi(\phi) Z(z), \\
R & =D(\rho, \lambda) J_{\rho}\left(\sqrt{\alpha^{2}+\lambda^{2}} r\right), \\
\Phi & =E(\rho, \lambda) \sin (\rho \phi)+C(\rho, \lambda) \cos (\rho \phi), \\
Z & =A(\rho, \lambda) \mathrm{e}^{-\lambda|z|}
\end{aligned}
$$

where $\lambda$ and $\rho$ are constants of separation, and the unknown functions $A, C, D, E$ are to be determined from the boundary conditions. The complete solution for $B$ is the integral over $\lambda$ ranging from 0 to infinity, and a sum over every $\rho$ (Arfken \& Weber 1995):

$$
\begin{aligned}
B(r, \phi, z)= & \sum_{\rho=0}^{\infty}\left[\int_{0}^{\infty} D(\rho, \lambda) J_{\rho}\left(\sqrt{\alpha^{2}+\lambda^{2}} r\right) A(\rho, \lambda)\right. \\
& \left.\times \mathrm{e}^{-\lambda|z|}(E(\rho, \lambda) \sin (\rho \phi)+C(\rho, \lambda) \cos (\rho \phi))\right] .
\end{aligned}
$$

For every component one finds two constants given through Fourier expansion. That is, extra boundary conditions are required. In our case for example, all components of the magnetic field in the accretion disk must be specified. However, the integral equations remain difficult to treat since the Bessel function is not linearly dependent on $\lambda$, and analytical solutions are not straightforward. We thus turn to the current-free case $(\alpha=0)$ where the equations can be simplified.

\section{Current-free configurations}

Here, $\boldsymbol{J}=\mathbf{0}$ is a necessary and sufficient condition to guaranty the existence of a scalar field $(\varphi)$ with the property, $\nabla \varphi=\boldsymbol{B}$. As can be seen in Appendix A this greatly simplifies our task of finding the initial configurations. We investigate two kind of current-free magnetic fields, with and without toroidal component $\left(B_{\phi}\right)$.

\section{1. $B_{\phi} \neq 0$}

Here we do not demand that the toroidal field is zero in the corona. We use the following boundary condition:

$B_{\phi, 0}=\left(b r^{\mu-1}\right) \times \sin (k \phi)$, where $B_{\phi, 0}$ is the toroidal magnetic field in the disk, and $b$ a normalization factor. The general solution for the scalar field is given in Appendix A where we also explain the choice of such a $\phi$ dependence. We find,

$$
\begin{aligned}
& \varphi(r, \phi, z)= q(\mu, k) b \cos (k \phi) \frac{r^{k}}{{\sqrt{r^{2}+|z|^{2}}}^{k-\mu+2}} \\
& \times{ }_{2} F_{1}\left(\frac{k-\mu+1}{2}, \frac{\mu+k}{2}, k+1, \frac{r^{2}}{r^{2}+|z|^{2}}\right), \\
& q(\mu, k)=2^{\mu-k+1} \frac{\Gamma\left(\frac{1}{2}(k+\mu+2)\right) \Gamma(k-\mu+2)}{\Gamma\left(\frac{1}{2}(k-\mu)\right) \Gamma(k+1)} \\
& \quad \text { for } 1-k<\mu<\frac{1}{2},
\end{aligned}
$$

where ${ }_{2} F_{1}$ is a hyper geometric function and $\Gamma$ the gamma function. The $k=2$ case, for example, is a simple solution. The corresponding configurations once implemented in the simulations remain stable in time. In the $B_{\phi} \neq$ 0 case, however, the corresponding jet simulations (including mass-load and disk rotation) are prone to pinch forces within few disk rotations. That is, the resulting dynamics is more complex, and the jet more difficult to track/investigate numerically. The $B_{\phi}=0$ configurations, also adopted in OPI\&II, turned out to be useful in many ways; it allows for instance to demonstrate that the jet collimation can result from the self-generated toroidal field. These we consider in details next.

\section{2. $B_{\phi}=0$}

In the current-free initial setup the differential equation for the scalar field of the magnetic field $(\varphi)$ reduces to the Laplace equation (since $\boldsymbol{\nabla} \cdot \boldsymbol{B}=0$ ):

$\nabla^{2} \varphi(r, \phi, z)=0$.

The corresponding scalar potential is found to be (Appendix A),

$$
\begin{aligned}
\varphi(r, \phi, z) & =2^{\mu} b z^{\mu} \frac{\Gamma\left(1+\frac{\mu}{2}\right) \Gamma(-\mu)}{\Gamma\left(1-\frac{\mu}{2}\right)} \\
& \times{ }_{2} F_{1}\left(\frac{1-\mu}{2},-\frac{\mu}{2}, 1,-\frac{r^{2}}{z^{2}}\right),-2<\mu<\frac{1}{2} .
\end{aligned}
$$

Solution above corresponds to the following boundary condition:

$B_{r, 0}=b r^{\mu-1}$,

where $B_{r, 0}$ is the radial component of the magnetic field in the accretion disk. Recall that we do not simulate the accretion disk (a fixed boundary in all of our simulations), but only the jet and the overlying corona $(z>0)$.

We examined configurations for 2 different values of $\mu$ (the radial dependence of the magnetic field); an open $(\mu=-1)$ and a closed $(\mu=-2)$ configuration. The closed configuration has the same radial dependence as a dipole field, which is expected (in first approximation) around 


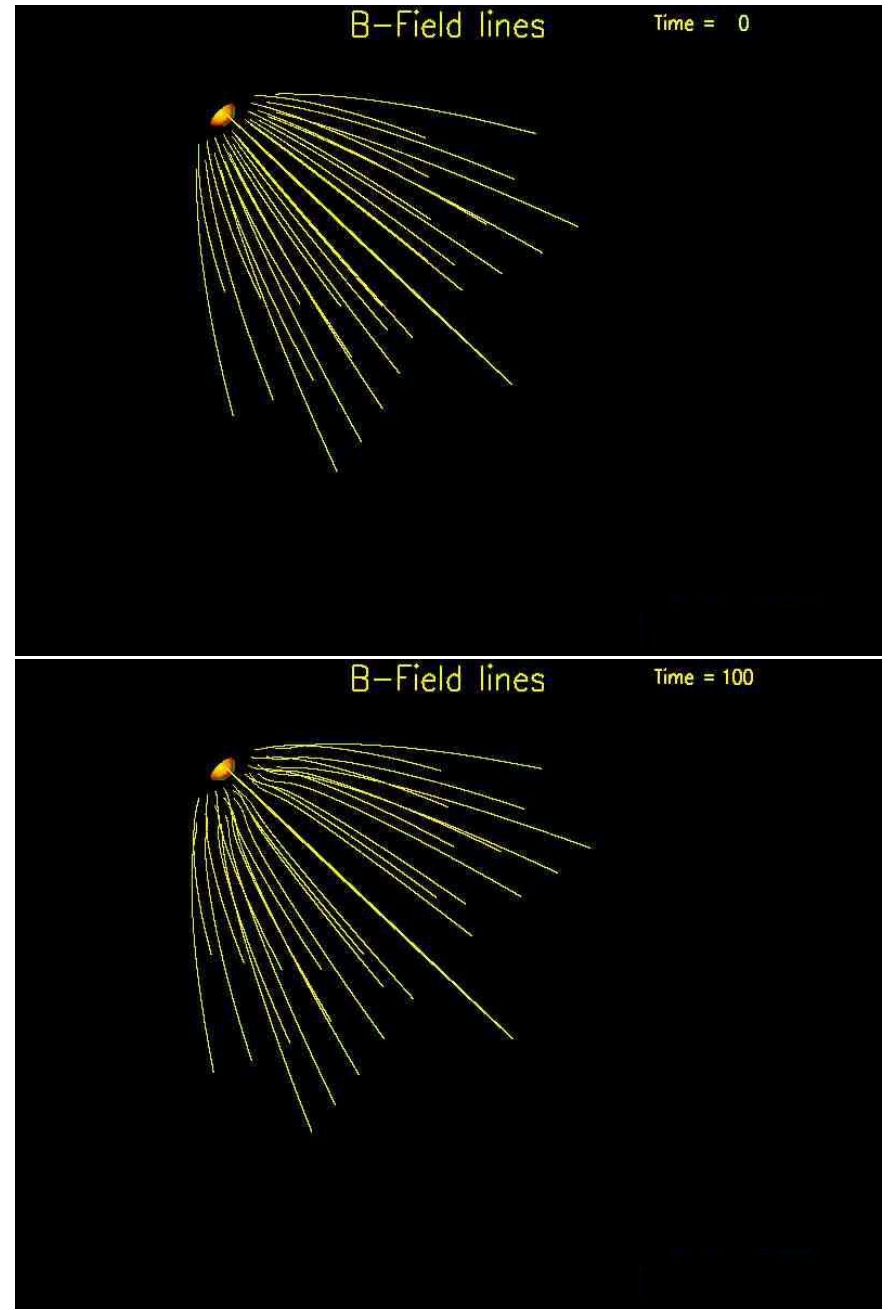

Fig. 1. Current-free solutions $\left(B_{\phi}=0\right)$ - the open case $(\mu=$ -1 ): shown here are the magnetic field lines at $\tau=0$ (top) and at $\tau=100$ (bottom). In this figure and the rest of figures, the blob in the center represents an iso-surface density around the central object (the proto-star is less than few pixels in size and cannot be seen in the figure). The rotation axis is shown crossing the iso-surface density into the central object and is in the plane of the paper. The accretion disk not shown here is to the far left and is perpendicular to the rotation axis.

stars however ours is strictly a solution of the disk boundary condition. The open configuration is the 3-D analogue/extension of the open configuration used in OPI. We implemented these 2 configurations in our initial setup and let them evolve in time after applying a small perturbation to the density. No mass injection and no disk rotation were set, and $v_{z}=v_{r}=v_{\phi}=0$ in the corona $(z>0.0)$. For the simulations we used the time-explicit Eulerian MHD-code ZEUS3D (Stone \& Norman 1992).

Cartesian coordinates, $(x, y, z)$, are used for all simulations. While being the natural system to use to avoid any directional biases, it does introduce some of its own problems not encountered in the 2-D cylindrically symmetric simulations. We refer the interested reader to OPC for the technical reasons underlying the choice of Cartesian
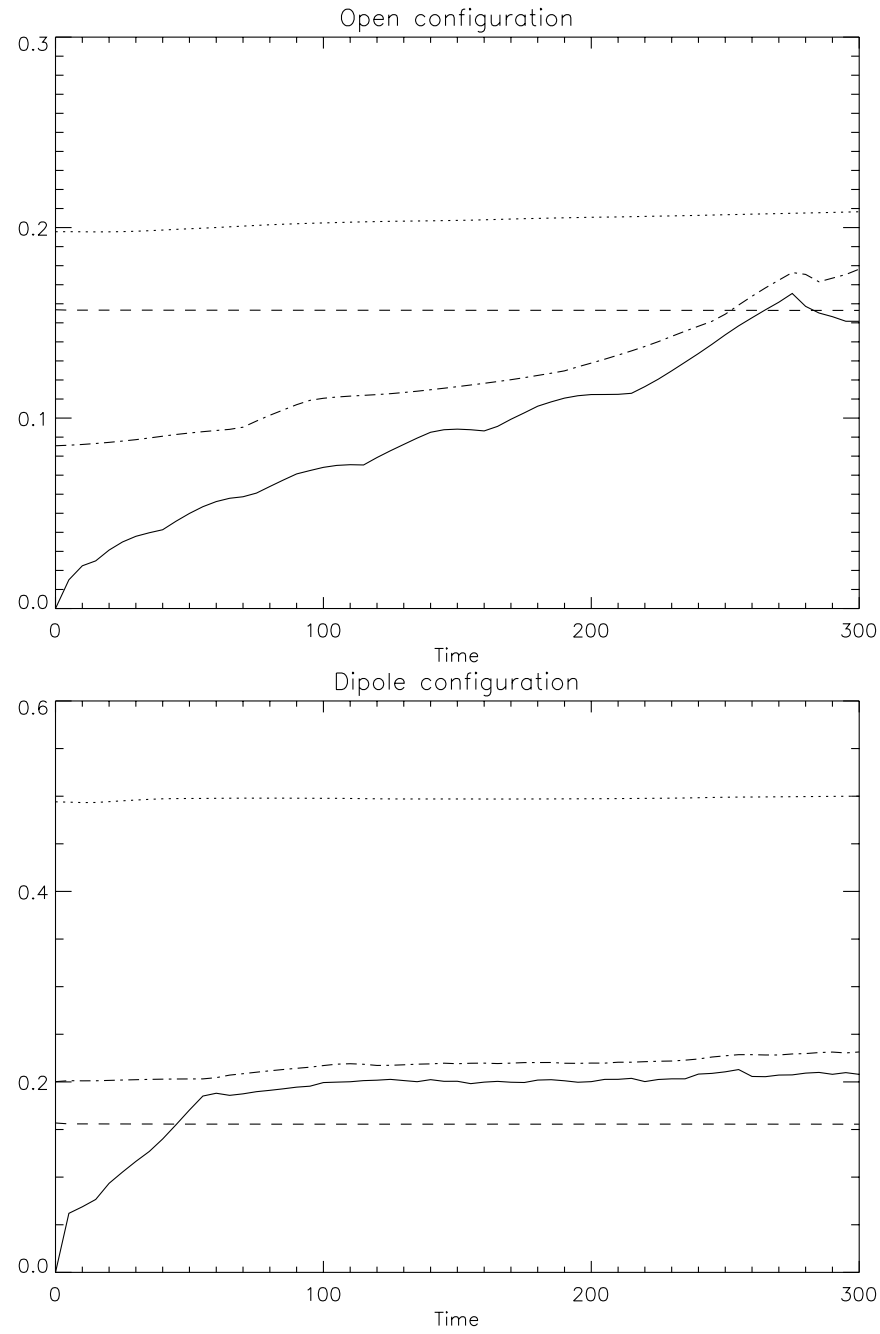

Fig. 2. Current-free solutions $\left(B_{\phi}=0\right)$ - the open case $(\mu=$ -1 ; top) and the dipole case $(\mu=-2$; bottom). Shown evolving in time are the maximum values for the velocity (solid line), the magnetic field strength (dotted line), and the density (dashed lines). The dot-dashed line shows the maximum speed for the propagation of Alfvén waves.

coordinates for such simulations. The disc is taken to lie along the $x-y$ plane, and the disc axis corresponds to the $z$-axis. In units of the inside radius of the disc, $r_{\mathrm{i}}$, the simulated region has dimensions $(-15:+15,-15:+15,0:+60)$, and is divided into $(95,95,120)$ uniform rectangular zones.

Figure 1 shows the magnetic field lines for the open configuration at time $\tau=0$ (in units of inner Kepler period) and at time $\tau=100$. The magnetic configuration changes only slightly in time and the generated currents are negligible. This is further demonstrated in Fig. 2 (top panel) where we show the time evolution of the maximum values for velocity, density and magnetic field strength. The induced velocity and slight variations in the magnetic field topology are signature of Alfvén waves which as expected have no dynamical effects on the coronal material. The solution stays force-free up to $\tau=300$ and no motion/jet is generated - as can 

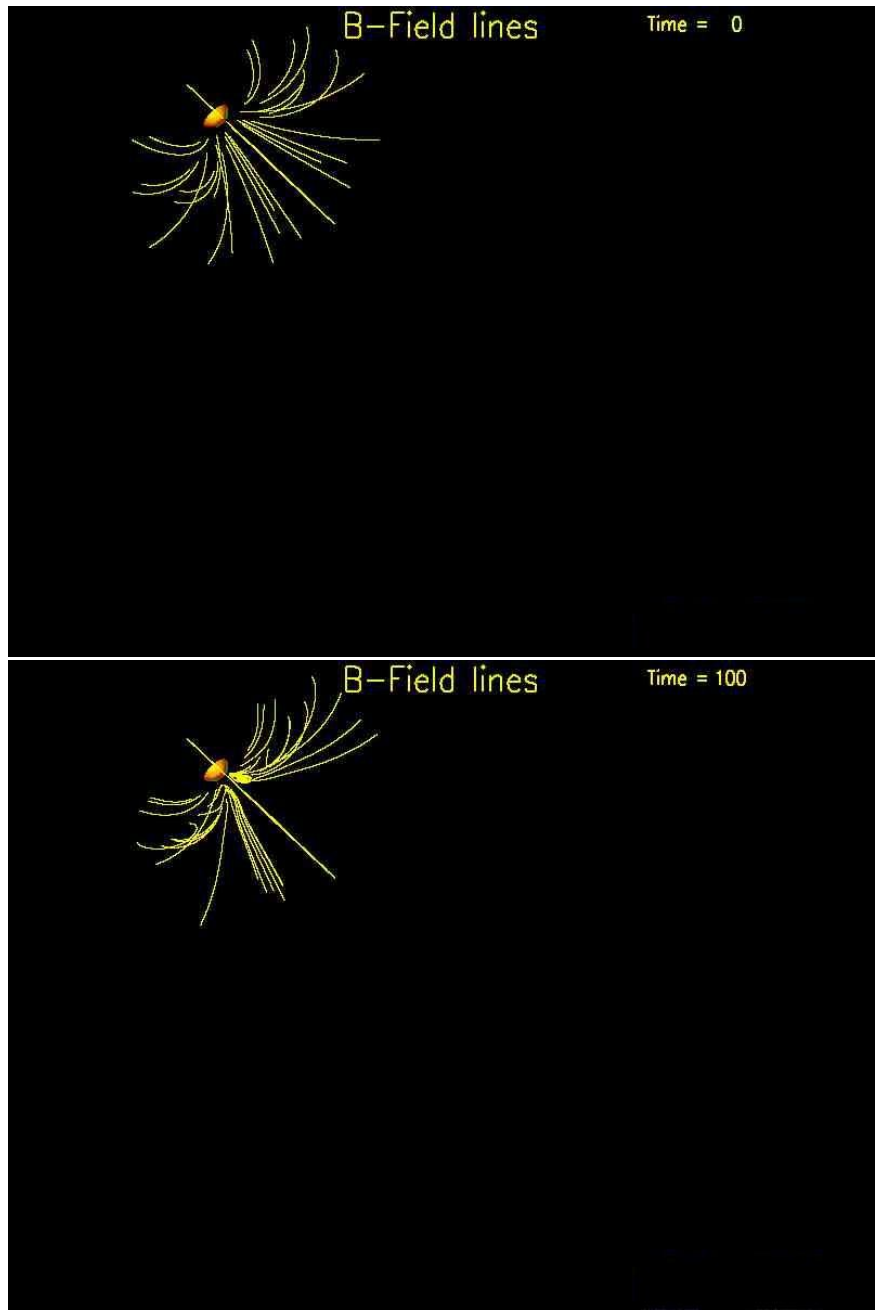

Fig. 3. Current-free solutions $\left(B_{\phi}=0\right)$ - the dipole case $(\mu=$ $-2)$. Shown are the magnetic field lines at $\tau=0$ (top) and at $\tau=100$ (bottom).

further be checked from the movies of the simulations (at http: //www.fys.ku.dk/ svart/Jets/). The closed configuration (lower panel in Fig. 2) is also stable in time although the induced errors are $20 \%$ larger than in the open case (Fig. 3). Here as well the initial hydrostatic balance is preserved up to $\tau=300$.

The simulations described above are easily implemented numerically and if it is left unperturbed, the corona will remain in perfect numerical balance to within machine round-off errors. In any case, when disk rotation and mass-load are taken into account, the small perturbations are quickly and completely washed out by the jet dynamics $(\tau \ll 50)$. The complete set of simulations with and without disk rotation and mass-loading can be visualized and compared at http://www.fys.ku.dk/ $\sim_{\text {svart/Jets/. }}$

\subsection{The JETSET tool}

We developed a tool, named JETSET, that generates initial states as described above. The JETSET main frame is shown in Fig. 4. Once the grid dimensions, the physical scales and the appropriate coronal and disk parameters have been specified, JETSET performs a NewtonRaphson method to find the correct density distribution while the corresponding magnetic field configuration is computed using the approach described in previous sections. The resulting Data (density, specific energies, velocity, and magnetic field) describing the initial setup is stored in an HDF (Hierarchical Data Format) file which can then be read by the user's code (such as ZEUS). The magnetic field lines can be visualized (see Fig. 4) as well as the density distribution around the central object (see Fig. 5). JETSET is available (down-loadable) at http://www.nordita.dk/ ouyed/JETTOOLS/. Included in the package are README and HELP files.

\section{Conclusion}

In this paper, we solved analytically for force-free solutions of magnetic configurations which can be implemented in 3-D simulations of astrophysical jets (disk winds). These configurations which thread the accretion disk and the corona, we showed, do not perturb the initial hydrostatic balance and are stable in time. While idealistic (developed for ease of implementation and computation), they constitute the first stage towards testing the effects of different magnetic configurations on the simulated jets in 3-D. Realistic configurations ought to reproduce basic features of astrophysical jets, such as their cylindrical shape, their knotty structure, and their stability.

Acknowledgements. We thank R. E. Pudritz and C. Rogers for helpful discussions.

\section{Appendix A: Current-free configurations}

\section{A.1. $B_{\phi} \neq 0$}

Here, the problem reduces to finding a general solution to Laplace's equation:

$\nabla^{2} \varphi=0$

Making a separation of variables in cylindrical coordinates,

$\varphi=R_{\varphi}(r) \Phi_{\varphi}(\phi) Z_{\varphi}(z)$

we find (Gradshteyn \& Ryznik),

$$
\begin{aligned}
\varphi(r, \phi, z) & =\int_{0}^{\infty} \sum_{\rho=0}^{\infty} D(\rho, \lambda) J_{\rho}(\lambda r) A(\rho, \lambda) \mathrm{e}^{-\lambda|z|} \\
& \times(E(\rho, \lambda) \sin (\rho \phi)+C(\rho, \lambda) \cos (\rho \phi)) \mathrm{d} \lambda
\end{aligned}
$$

where $\lambda$ and $\rho$ are constants of separation, and $J_{\rho}(\lambda r)$ the Bessel function. The unknown functions $A, C, D, E$ which can in fact be combined in two constants (see below) within the Fourier space are to be determined from the boundary conditions. Equation (A.3) is a superposition of the solutions to $R_{\varphi}, \Phi_{\varphi}$ and $Z_{\varphi}$. It shows that the 


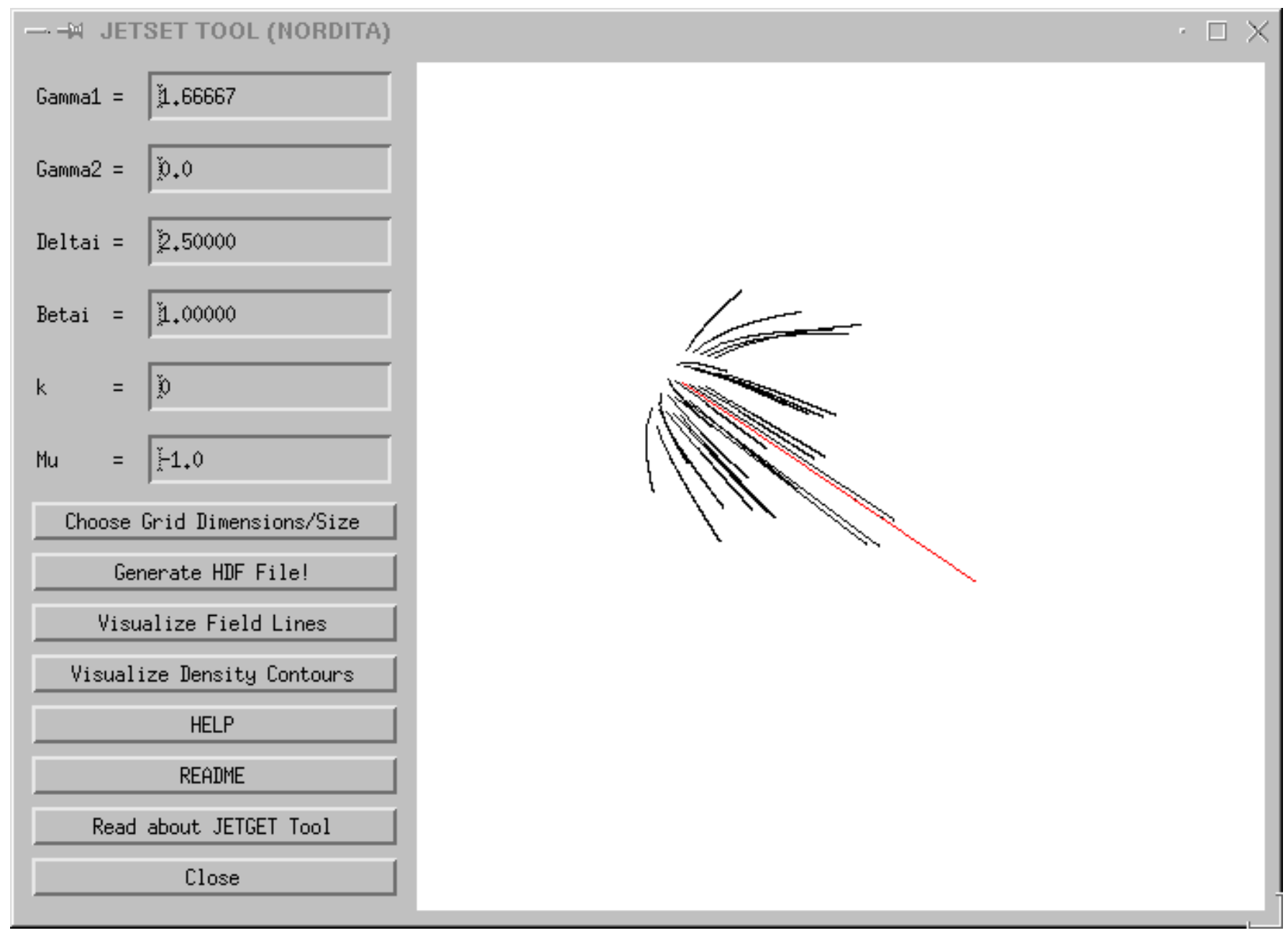

Fig. 4. JETSET TOOL: JETSET sets initial states for astrophysical jet simulations in 3-D. The initial set up consists of a corona in hydrostatic balance around a central object with current-free magnetic fields threading through the corona and the underlying accretion disk (fixed boundary). JETSET solves for the correct density distribution around the central object and the appropriate current-free magnetic field configuration once the two parameters, $k$ and $\mu$, are specified (see text). The coronal material might consist of one pressure component $\left(\gamma_{1}=5 / 3, \gamma_{2}=0\right)$ or two-pressure component $\left(\gamma_{1} \neq 0, \gamma_{2} \neq 0\right.$, where $\gamma_{1}$ and $\gamma_{2}$ are the corresponding adiabatic indices). The initial magnetic field configuration can be visualized and saved into a file. Figures similar to the upper panels in Figs. 1 and 2 can be generated (and saved into files) by JETSET as evident from the renderer to the right. Further details on JETSET can be found in the README and HELP files included in the JETSET package which can be down-loaded at http://www.nordita.dk/ ouyed/JETTOOLS/.

constants may be determined from a single boundary condition, for example the configuration of the toroidal magnetic field in the disk $B_{\phi, 0}=B_{\phi}(r, \phi, 0)$.

We now look at the $B_{\phi}=\left.\nabla \varphi\right|_{\phi}$ component:

$$
\begin{aligned}
B_{\phi}=\sum_{\rho=0}^{\infty} & {\left[\cos (\rho \phi)\left(\rho \int_{0}^{\infty} S_{1, \rho}(\lambda) \mathrm{e}^{-\lambda|z|} J_{\rho}(\lambda r) \mathrm{d} \lambda\right)+\right.} \\
& \left.\sin (\rho \phi)\left(-\rho \int_{0}^{\infty} S_{2, \rho}(\lambda) \mathrm{e}^{-\lambda|z|} J_{\rho}(\lambda r) \mathrm{d} \lambda\right)\right]
\end{aligned}
$$

where $S_{1, \rho}(\lambda)=D(\rho, \lambda) E(\rho, \lambda) A(\rho, \lambda)$ and $S_{2, \rho}(\lambda)=$ $D(\rho, \lambda) C(\rho, \lambda) A(\rho, \lambda)$.

Equation above is simply a Fourier expansion in the toroidal dependence of the magnetic field. The integrals before the trigonometric functions are the Fourier coefficients; so we may write:

$$
\begin{aligned}
& \int_{0}^{\infty} S_{1, \rho}(\lambda) \lambda \mathrm{e}^{-\lambda|z|} J_{\rho}(\lambda r) \mathrm{d} \lambda=\frac{1}{\pi \rho} \int_{0}^{2 \pi} B_{\phi} \sin (\rho \phi) \mathrm{d} \phi \\
& -\int_{0}^{\infty} S_{2, \rho}(\lambda) \lambda \mathrm{e}^{-\lambda|z|} J_{\rho}(\lambda r) \mathrm{d} \lambda=\frac{1}{\pi \rho} \int_{0}^{2 \pi} B_{\phi} \cos (\rho \phi) \mathrm{d} \phi,
\end{aligned}
$$

where the right hand side is the expressions for the Fourier coefficients. Note that $S_{1, \rho}(\lambda)$ and $S_{2, \rho}(\lambda)$ are the Hankel transforms of the Fourier components of $B_{\phi}(r, \phi, 0)$. That is, for any given toroidal configuration of the magnetic field in the disk, $S_{1, \rho}(\lambda)$ and $S_{2, \rho}(\lambda)$ are given by equations above thus providing a simple procedure for determining the scalar field.

Finally, note that the simplest magnetic configuration possible is given by $S_{\rho}(\lambda)=0 \forall \rho \neq k$ which gives us the boundary condition investigated in this paper 

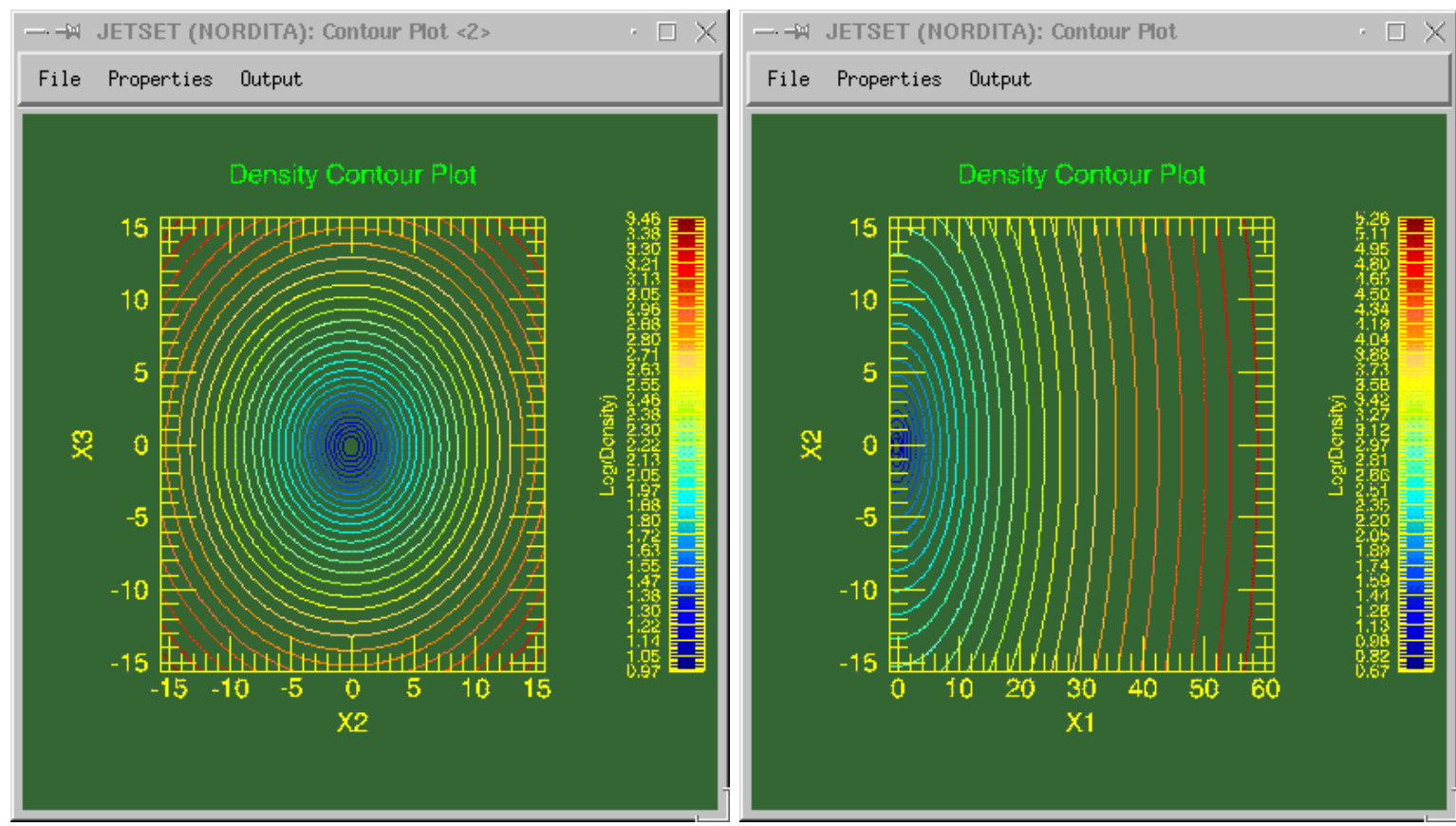

Fig. 5. JETSET TOOL - Density contours as generated by JETSET for a single pressure component corona with $\gamma_{1}=5 / 3$. The left panel is in a plane parallel to the disk surface while the panel to the right shows the density contours in a plane perpendicular to the disk surface and containing the disk rotational axis.

$B_{\phi, 0}=\left(b r^{\mu-1}\right) \times \sin (k \phi)$ (including the $k=0$ case with no toroidal dependence); $b$ is a scaling factor.

\section{A.2. $B_{\phi}=0$}

The corresponding scalar field is obtained by cancelling the $\phi$ dependence in (A.3). The components $B_{r}$ and $B_{z}$ are then direct derivative of $\varphi$. For example, one can show that

$B_{z}=\int_{0}^{\infty} S(\lambda) \lambda J_{0}(\lambda r) \mathrm{e}^{-\lambda|z|} \mathrm{d} \lambda$,

where $S(\lambda)$ is the Hankel transform of $B_{z}(r, \phi, 0)$.

\section{References}

Arfken, G. B., \& Weber, H. J. 1995, in Mathematical methods for physicists (Academic Press, fourth edition)

Blandford, R. D., \& Payne, D. R. 1982, MNRAS, 199, 883

Gradshteyn, I. S., \& Ryznik, I. M. 1965, in Table of Integrals, Series and Products (Academic Press Inc., fourth edition, New York)

Ouyed, R., \& Pudritz, R. E. 1997a, ApJ, 482, 712 (OPI)

Ouyed, R., \& Pudritz, R. E. 1997b, ApJ, 484, 794 (OPII)

Ouyed, R., Pudritz, R. E., \& Clarke, D. A. 2001, ApJ, submitted (OPC)

Stone, J. M., \& Norman, M. L. 1992, ApJS, 80, 753 